\title{
Revocation for Certificateless Authentication in VANETs
}

\author{
Pino Caballero-Gil, Francisco Martín Fernández, Cándido Caballero-Gil \\ Department of Computer Engineering \\ University of La Laguna \\ San Cristobal de La Laguna, Spain
}

\begin{abstract}
This paper proposes a new way to deal with the problem of revocation of malicious users in vehicular ad-hoc networks. In particular, the proposal is designed to be used for identity-based authentication, which allows taking advantage of the efficiency and safety of certificateless authentication. In order to improve the performance of revocation lists, this paper proposes the use of a data structure based on authenticated dynamic hash $k$-ary trees and the frequency with which revoked pseudonyms are queried. Knowledge about query frequencies of revoked pseudonyms provides a more efficient access to the most popular revoked pseudonyms to the detriment of revoked pseudonyms that are the least queried. Accordingly, the proposal is especially useful in urban environments where there are vehicles that spend more time on the road than others.
\end{abstract}

\section{Introduction}

Authentication is a critical piece in the security of wireless communications where the existence of effective mechanisms for revocation of users is required. On the one hand, it is necessary to optimize the authentication process so that legitimate and honest nodes can make use of all network services. On the other hand, the authentication process must be useful to detect and exclude the malicious nodes, in order to ensure network reliability.

A major problem when communication security is based on public-key cryptography is to ensure that any particular public key is authentic and valid. The traditional approach to this problem is through public-key certificates emitted by a Public Key Infrastructure (PKI), in which a Certificate Authority (CA) certifies ownership and validity of public-key certificates. This solution presents many difficulties because the issues associated with certificate management are quite complicated and expensive. The so-called Identity Based Cryptography (IBC), where each user's public key is his/her public IDentity (ID), represents a different approach because the need for public key certificates is eliminated.

Efficiency is a key feature in revocation when public-key cryptography is used because private keys may become compromised. This problem has been traditionally solved through a centralized approach based on the existence of a Trusted Third Party (TTP), which is usually a CA distributing the socalled Certificate Revocation Lists (CRLs) that can be seen as blacklists of revoked certificates. Moreover, in order to achieve a more efficient management of certificate revocation, a different approach based on hash trees as Authenticated Data Structures (ADS) has been proposed.

A proposal to prevent adverse circumstances on the roads and to achieve more efficient traffic management corresponds to the idea of Vehicular Ad-hoc NETworks (VANETs). They can be seen as self-organizing networks built up from moving vehicles that communicate with each other. In particular, these networks are considered an emerging research area of mobile communications because they offer a wide variety of possible applications, ranging from as aforementioned road safety and transport efficiency, to commercial services, passenger comfort, and infotainment delivery. Furthermore, VANETs can be seen as an extension of mobile ad-hoc networks where there are not only mobile nodes, named On-Board Units (OBUs), but also static nodes, named Road-Side Units (RSUs). The so-called Intelligent Transportation System (ITS) includes two types of communications: between OBUs and between OBUs, and RSUs [5]. IEEE 802.11p amendment to IEEE 802.11, which adds a vehicular communication system is the basis for both the European standard for ITS, called ITS-G5, and its American counterpart, called Wireless Access in Vehicular Environment (WAVE).

The protection of the security of communications is more difficult due to the open broadcasting of wireless communications and the high-speed mobility of vehicles in VANETs. In these networks, any malicious misbehaving user that can inject false information, or modify/replay any previously disseminated message, could be fatal to the others. Therefore, within the family of standards for vehicular communications IEEE 1609 based on the IEEE $802.11 \mathrm{p}$, the standard 1609.2 deals in particular with the issues related to security services for applications and management messages. This standard describes the use of PKIs, CAs and CRLs, 
and implies that in order to revoke a vehicle, a CRL has to be issued by the CA to the RSUs, who are in charge of sending this information to the OBUs. In particular, the IEEE 1609.2 standard proposes both broadcast authentication and non-repudiation through the use of the Elliptic Curve Digital Signature Algorithm (ECDSA). However, the verification of each signature using ECDSA means a high computational cost. On the one hand, according to these standards, each vehicle is assumed to have a pair of keys: a private signing key and a public verification key certified by the $\mathrm{CA}$; and any VANET message must contain: a timestamp with the creation time, the sender's signature, and the sender's public-key certificate. On the other hand, the socalled Dedicated Short-Range Communications (DSRC), devoted specifically designed for automotive use, defines that vehicles regularly exchange with nearby vehicles beacons containing sender information such as location and speed, because the information of these beacons is very useful for many VANET applications, such as cooperative collision warning.

Each OBU can get multiple certified key pairs and use different public keys each time certified in order to protect privacy in VANETs. These public keys are linked to pseudonyms that allow preventing location tracking by eavesdroppers. Therefore, once VANETs are implemented in practice on a large scale, their size will grow rapidly due to the increasing number of OBUs and to the use of such multiple pseudonyms. Thus, it is foreseeable that if CRLs are used, they will grow up to become very large and unmanageable. Moreover, this context can bring a phenomenon known as implosion request, consisting of many nodes who synchronously try to download the CRL during its updating, producing a longer latency in the process of validating a certificate due to serious congestion and overload of the network.

This work proposes the use of IBC to achieve certificateless and cooperative authentication in VANETs. It also introduces a Huffman k-ary hash tree as an ADS for the management of pseudonym revocation. By using this $\mathrm{ADS}$, the process of query on the validity of public pseudonyms will be more efficient because OBUs will send queries to RSUs, who will answer them on behalf of the TTP. In this way, at the same time this TTP will no longer be a bottleneck and OBUs will not have to download any entire revocation list. Instead of that, they will have to manage hash trees where the leaf nodes contain revoked pseudonyms. In particular, in order to provide the shortest paths in the tree for the revoked pseudonyms that are the most queried by network users, the use of k-ary Huffman trees is proposed so that we can take advantage of the efficiency of the Huffman algorithm.
This paper is organized as follows. Section 2 presents a review of state of the art. Concepts and notation used in the proposed authentication scheme based on the combination of IBC and Huffman k-ary hash trees are introduced in Section 3. Section 4 summarizes the main ideas of the proposal. Finally, Section 5 discusses some conclusions and open problems.

\section{State of the Art}

One of the fundamental protocols for information security is the use of public-key cryptography [2]. For instance, an aforementioned, the family of standards IEEE 1609 describes the use of PKI in VANETs. In particular, the work [9] analyzes a proposal for the use of a PKI to protect messages and mutually authenticate entities in VANETs. The paper is a continuation of that work because it describes a PKI-based security protocol where each vehicle preloads anonymous public/private keys and a TTP stores all the anonymous certificates of all the vehicles [19]. However, the certificate management process of this scheme is less efficient.

A well-known solution for strong authentication in VANETs also based on a PKI uses the signature of each message [11]. However, the use of a traditional approach to PKIs may fail to satisfy the real time requirement in vehicular communications because according to the DSRC protocol, each OBU will periodically transmit beacons so even in a normal traffic scenario, it is a very rigorous requirement to deploy an authentication scheme that allows at the same time efficient revocation of invalid public keys, and efficient use of valid public keys.

The Online Certificate Status Protocol (OCSP) is a revocation method that involves a multitude of validation agents that respond to client queries with signed replies indicating the current status of a target certificate. This explicit revocation method has an unpleasant side effect because it divulges too much information. Since validation agents constitute a global service, they must involve enough replication to handle the load of all validation queries, what means that the method is either insecure or expensive because the signature key must be replicated across many servers.

The Certificate Revocation Tree (CRT) is a solution proposed as an improvement for OCSP involving a single highly secure entity that periodically posts a signed CRL like data structure to many insecure validation agents so that users query these agents [15]. In CRTs, the leaf nodes are statements concerning revoked certificates, and the CA signs the root. By using CRTs, the responder can prove the status of any certificate by showing the path from the root to the leaf node without signing the response, because the signatures of any leaf node 
are identical, and given by the signature contained in the root. Therefore, there is no need to trust the responder. The certificateless proposal described here is based on this idea.

The ADS proposed is a Merkle hash tree where the leaf nodes represent revoked certificates sorted by serial number [15] [16]. A client sends a query to the nearest agent, which produces a short proof that the target certificate is (or not) on the CRT. Jakobsson introduces several methods to traverse Merkle trees allowing time space trade-offs [12]. Other ADSs based on multi-dimensional tree structures are studied to support efficient search queries, allowing the retrieval of authenticated certificates from an untrusted repository used for dissemination by various credential issuers [17]. Besides, many tree-balancing algorithms have been proposed in the bibliography for hash trees [4]. For instance, AVL trees are balanced by applying rotation, B-trees are balanced by manipulating the degrees of the nodes, and 2-3 trees contain only nodes with at least 2 and at most 3 children. However, in the particular application of public-key revocation, balanced trees do not necessarily minimize communication.

Each time a certificate is revoked, another interesting problem with CRTs appears because the whole tree must be recomputed and restructured. Skip-lists proposed can be seen as a natural and efficient structure for the purpose of reducing communication by balancing the CRT [7] [8]. However, for problems such as insertion of new leaf nodes, these are not good solutions.

Widely used hash functions are typically used on hash trees. This work uses SHA-3, which is a cryptographic hash function recently selected as the winner of the NIST hash function competition based on the Keccak function and a sponge construction [1].

In order to solve the problem caused by the management of valid public-key certificates, the work proposes the idea of an identity-based cryptosystem in which arbitrary strings can act as public keys so that there is no need for public-key certificates [20]. The first practical identity-based encryption scheme was described by Boneh using a bilinear map [3]. Weil and Tate pairings on elliptic curves are the most efficient ways of constructing such bilinear maps [14]. For the implementation of the identity-based authentication in the proposal here described the Tate pairing was used.

\section{Fundamentals}

\subsection{ID-Based Cryptography}

The idea of IBC and, in particular, of IdentityBased Signature (IBS) based on that the public identity ID of the signer can be used as verification key of a received signature, what avoids the need of any public-key certificate. In our scheme, such an identity is a public pseudonym $\mathrm{Pj}$ sent by the signer node together with the signed message. In the used ID-based system, each node has to receive all the signing private keys $\mathrm{PrPj}$ linked to all its pseudonyms $\mathrm{Pj}$ from a TTP, because it cannot generate them by itself. In particular, a TTP, called in IBC the Private Key Generator (PKG), is in charge of computing and delivering to each node via a confidential channel, the signing private keys linked to each of its pseudonyms. On the other hand, the PKG publishes a master public key $\mathrm{MPu}$ and retains the corresponding master private key MPr. Thus, given the master public key $\mathrm{MPu}$, any party will be able to compute the public key $\mathrm{PuPj}$ corresponding to any pseudonym $\mathrm{Pj}$ by combining it with $\mathrm{MPu}$. In order to use the corresponding private key, the node authorized to use a pseudonym must have received it from the $\mathrm{PKG}$, which uses the master private key MPr to generate all the private keys corresponding to all the pseudonyms. Thus, the main algorithms in the proposed IBS are as follows:

- Setup: The PKG randomly picks its master private key MPr, and therefore computes and publishes its master public key MPu.

- Extraction: For each pseudonym Pj, the PKG uses its master private key $\mathrm{MPu}$ to compute the corresponding private key $\mathrm{PrPj}$ and all pairs $(\mathrm{Pj}$, $\mathrm{PrPj}$ ) are sent securely from the PKG to the corresponding owner.

- Signature: A signer node uses its private key PrPj to compute the signature of a message $\mathrm{M}$, and sends openly both the computed signature $\operatorname{PrPj}(\mathrm{M})$ and its pseudonym $\mathrm{Pj}$.

- Verification: A node that receives a signed message and corresponding pseudonym $(\operatorname{PrPj}(\mathrm{M}), \mathrm{Pj})$ uses $\mathrm{MPu}$ and $\mathrm{Pj}$ to compute $\mathrm{PuPj}$ and verify the signature $\operatorname{PrPj}(\mathrm{M})$.

Note that no new ID-based cryptosystem is described in this paper because it is out of its scope. The ID-based system that has been implemented in the proof of concept prototype is the Boneh-Franklin scheme, which uses a bilinear pairing over elliptic curves and bases its security on the Bilinear DiffieHellman problem [3].

The ID-based system used, is built from a bilinear map e: $\mathrm{G} 1 \times \mathrm{G} 1 \rightarrow \mathrm{G} 2$ between two groups G1 and G2 so that according to the bilinearity of e: $\mathrm{e}(\mathrm{aP}, \mathrm{bQ})=\mathrm{e}(\mathrm{P}, \mathrm{Q}) \mathrm{ab}$ for all $\mathrm{P}, \mathrm{Q} \in \mathrm{G} 1$ and $\mathrm{a}, \mathrm{b} \in \mathrm{Z}$. Specifically, an ID-based system can be built from a bilinear map e if and only if a variant of the Computational Diffie-Hellman problem in G1 is hard. The considered Bilinear Diffie-Hellman problem in $\mathrm{G} 1$ is defined as follows: Given $\mathrm{P}, \mathrm{aP}$, $\mathrm{bP}, \mathrm{cP}$, compute $\mathrm{e}(\mathrm{P}, \mathrm{P}) \mathrm{abc}$, where $\mathrm{P} \in \mathrm{G} 1$ and $\mathrm{a}, \mathrm{b}, \mathrm{c}$ $\epsilon$ Z. In particular, the used bilinear pairing $\mathrm{e}$ is 
described for an elliptic curve E defined over some field $\mathrm{K}$, so it maps a pair of points of $\mathrm{E}$ to an element of the multiplicative group of a finite extension of $\mathrm{K}$.

The first satisfactory version of the BonehFranklin scheme was based on the Weil pairing [3]. However, the scheme implemented in this work uses the Tate pairing because this is considered the most convenient bilinear function for the Boneh-Franklin scheme in terms of computational cost. In particular, the implementation of the proposal includes the use of Miller's algorithm to compute the Tate pairing [17].

In IBC, just a few works exist on revocation mechanisms. However, proposals of new solutions to provide efficient mechanisms for key revocation are neccesary. Here we propose a scheme to manage revoked pseudonyms in IBC-based VANETs, built on the idea of revocation hash trees.

\subsection{Nomenclature}

The tree-based model described in this paper is based on the following notation:

$h(\ldots)$ : Hash function used to define the revocation tree.

$h\left(A_{0}\left|A_{l}\right| \ldots\right)$ : Digest obtained with the hash function $\mathrm{h}$ applied on the concatenation of the inputs $\mathrm{A}_{\mathrm{i}}, \mathrm{i}=0,1 \ldots$

$D(\geq 1)$ : Depth of the hash tree.

$t$ : total number of revoked pseudonyms.

$R P_{j}(j=1,2, \ldots, t): \mathrm{j}$-th Revoked Pseudonym.

$N_{0}$ : Root Node of the hash tree.

$N_{\text {path }}$ : Node of the hash tree. The path indicates branches that you have to take to get from the root node to the leaf node representing.

$k$ : Maximum number of children for each internal node in the hash tree.

$f(\ldots)$ : Keccak function used in SHA-3.

$n$ : Bit size of the digest of $h$.

$s$ : Bit size of the input to $\mathrm{f}$.

$r$ : Bit size of the input blocks for $\mathrm{h}$ after padding.

$l$ : Bit size of the output blocks that build the digest of $\mathrm{h}$, which is here assumed to be lower than $\mathrm{r}$.

\subsection{Applying Huffman Codes}

Redesigning the structure of conventional revocation lists, to improve efficiency, has been a recurring theme in recent years in the investigation of VANETs. Indeed, some authors have proposed the use of particular ADSs such as Merkle trees, Huffman Merkle trees and skip lists [6] [13] [18]. However, to the best of our knowledge no previous work has described in detail the use of Huffman kary trees as hash trees for revoked pseudonym management.

In general, a hash tree is a tree structure whose nodes contain digests that can be used to verify larger pieces of data. The leaf nodes in a hash tree are hashes of data blocks while nodes further up in the tree are the hashes of their respective children so that the root of the tree is the digest representing the whole structure. Most implemented hash trees require the use of a cryptographic hash function $h$ in order to prevent collisions.

Like most hash trees, the Merkle tree is a binary tree, so each internal node $\mathrm{Nij}$ is the hash value of the concatenation of its two children: $\mathrm{Nij}=\mathrm{h}(\mathrm{Ni}-1,0 \mid \mathrm{Ni}-1,1)$, where $\mathrm{i}$ is the depth of node in the tree.

On the contrary, this work proposes the use of a more general structure known as k-ary tree, which is a rooted tree in which each node has no more than $\mathrm{k}$ children, and each internal node is obtained by hashing the concatenation of all the digests contained in its children. Specifically, we propose the use of a Huffman k-ary tree in which leaf nodes are ordered from left to right, based on which revoked pseudonyms the most queried. Thus, we propose the introduction of the combination of both concepts of Huffman coding and k-ary trees applied to trees based revocation.

Huffman coding is an algorithm used for data compression [10]. The term refers to the use of a table of variable length codes for encoding certain symbols, where the table has been filled in a specific manner based on the best probability estimated of occurrence of each possible value of the symbol. Our proposal is to bring that idea to the CRLs, and allocate shortest paths in the tree to the revoked pseudonyms that are most queried. Thus, the tree will be built according to the consulted frequency in which the pseudonyms are by users of VANETs. In this way, the query of the consulting most revoked pseudonyms will be which more efficient.

Each node $\mathrm{N}_{\text {path }}$ of our Huffman hash tree is given by a hash value. For each node $\mathrm{N}_{\text {path }}$, path is defined by the path from the root $\mathrm{N}_{0}$ to the node $\mathrm{N}_{\text {path }}$ (see Figure 1). The length of the path, given by the number of levels in the tree used in it, is related to the number of hash applications that are needed to represent the leaf node that corresponds to a revoked certificate. When a revoked node is very consulted, it will appear higher up in the tree to have a much shorter path. This improves the efficiency of both the search node in the tree, and the path proves of revocation because the size of the paths is greatly reduced.

The authenticity of the used hash tree structure is guaranteed thanks to the TTP signature of the root $\mathrm{N}_{0}$. When a RSU answers to an OBU about a query on a pseudonym, it proceeds in the following way. If it finds the digest of the pseudonym among the leaf nodes of the tree, which means that it is a revoked pseudonym, the RSU sends to the OBU the route between the root and the corresponding leaf node, along with all the siblings of the nodes on this path. 
After checking all the digests corresponding to the received path, and the TTP signature of the root, the OBU gets convinced of the validity of the received evidence on the revoked pseudonym. Conceptually, thanks to the proposal of using a Huffman tree, queries regarding the most usually queried certificates involve less data transmission and computation.

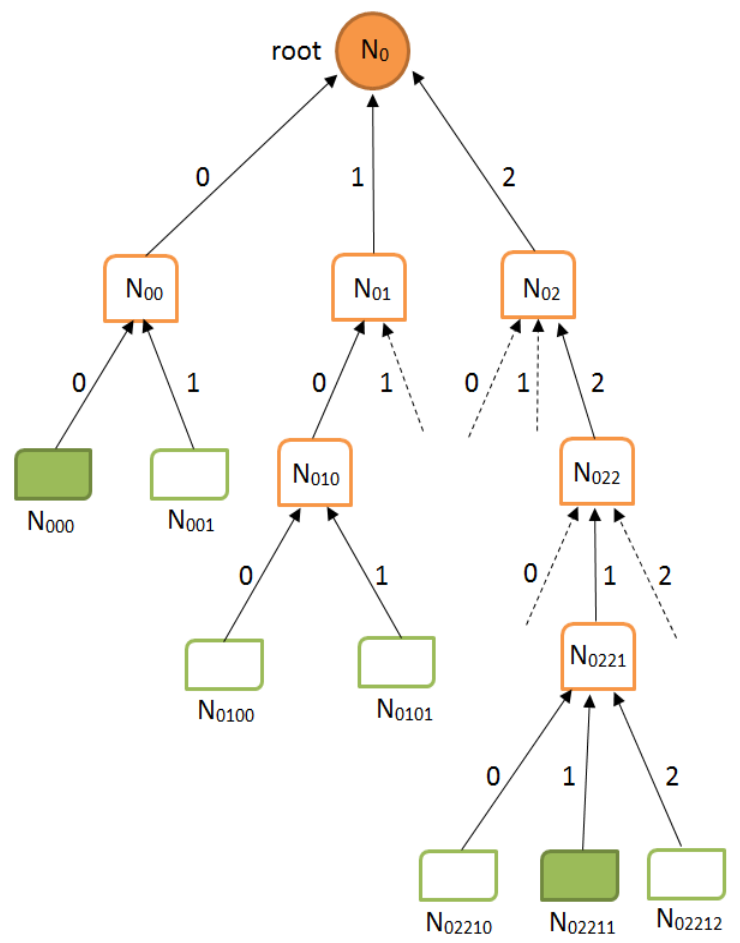

Figure 1. Revocation Path on Huffman 3-ary Tree

\subsection{Tree Construction}

The concept of using the Huffman algorithm applied to CRLs is planned to optimize the query time and computation about revoked pseudonyms in VANETs. Generally, vehicles that spend more time on the roads are those that are more likely to communicate with other vehicles. Typical systems based on CRLs do not take into account this factor, so the average cost of finding any revoked pseudonym is the same. However, the general cost can be optimized by assigning less deep positions in the hash tree, to the most queried pseudonyms corresponding to the vehicles that stay longer on the road.

For this reason, the proposal includes the assumption that the RSU counts the number of queries that are performed for each revoked pseudonym. During the update of the tree the nodes are rearranged based on the new frequencies. Furthermore, taking into account the type of vehicle we can see that public vehicles (buses, taxis, etc.) are more likely to be among the most queried ones because they spend much time on the road.

In order to build the tree, the first thing to consider is how many children per node is allowed to have a maximum and minimum. This parameter defines the $\mathrm{k}$ of the $\mathrm{k}$-ary tree to be built. If this $\mathrm{k}$ is equal to 2 , we get the typical binary Huffman tree. The proposed system allows other values for $\mathrm{k}$, such as 3, 4, 5, etc. Thus, if we propose a k-ary tree with a maximum of 5 children per node, we have a Huffman 5-ary like the one Figure 2.

Once we know how many children per node are allowed as maximum, what we do is to create the Huffman tree so that internal nodes are assigned from the query frequencies calculated by the RSU. Whenever $\mathrm{k}$ revoked pseudonyms are grouped in an internal node, this node is created with the sum of the frequencies of its children. In this way, the tree is constructed by grouping, first all revoked pseudonyms that are less consulted on new internal nodes, and so on to leave the most queried revoked pseudonyms in top positions in the tree. Thus, the search of these nodes is much faster and the route to the root node is much shorter.

In order to learn how to find a node in the tree, a hash table is used to map each revoked pseudonym with the exact path that defines the tree. Thus, if for example we have a Huffman 3-ary tree, we have that a node pseudonym $\mathrm{N}_{02211}$ has a path in the tree $[2,2,1,1]$ (see Figure 1), what means that from the root node we have to go through the branches starting by the branch 2 , then the $2 \ldots$ and so on to the internal node that is linked to it and opt for the branch 1 to get to it.

\section{Proposed Scheme}

In the scheme proposed in this work, a node does not need any certificate to prove the binding to its public key. Instead of that, an ID-based authentication scheme and revocation trees are used. We consider the following basic authentication architecture, which includes three main parties:

- TTP: This entity acts as key distribution center because it is responsible for generating and assigning related parameters for VANET nodes, and for revoking pseudonyms of misbehaving OBUs and public keys of misbehaving RSUs. 


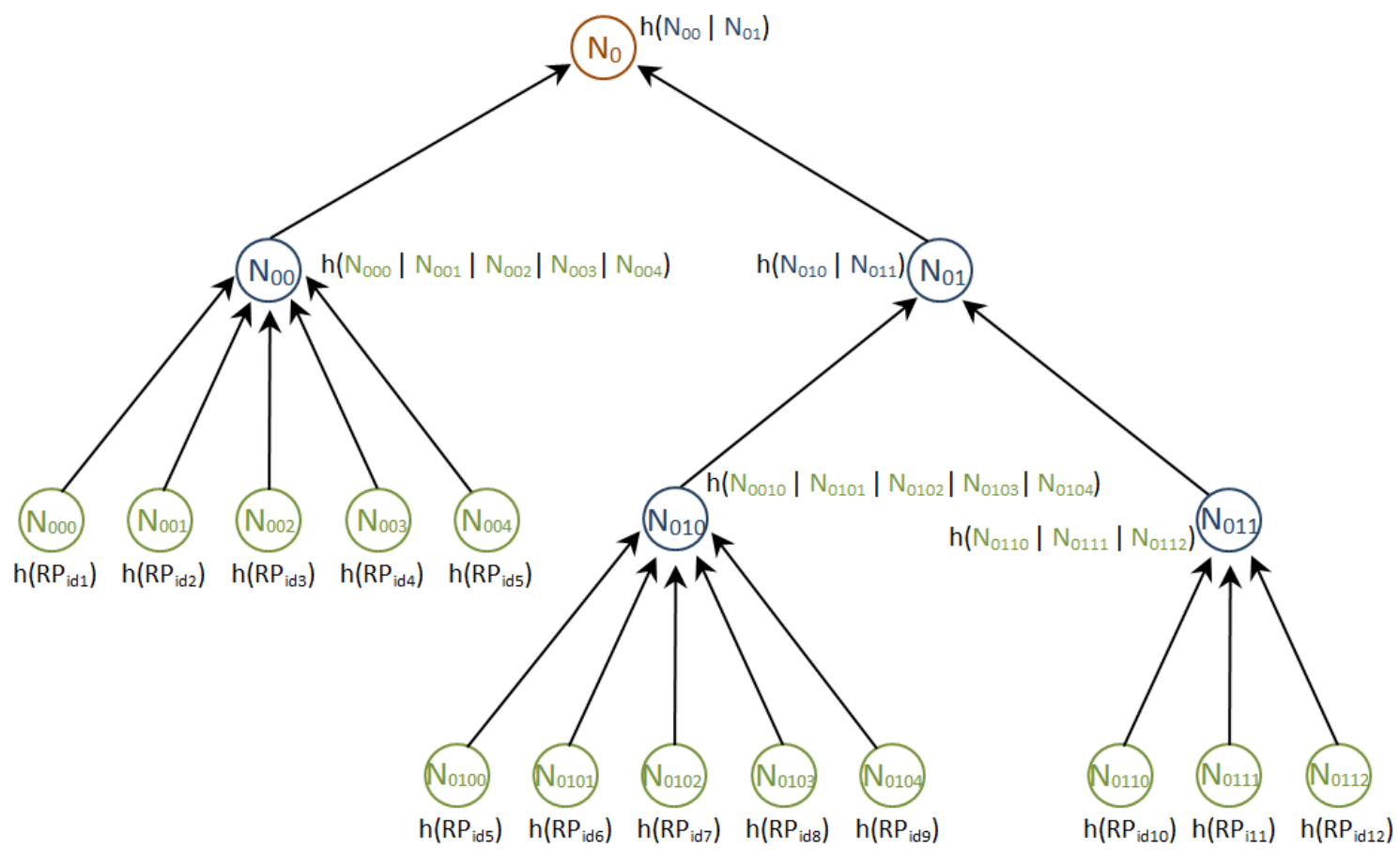

Figure 2. Hash Tree Based on Huffman 5-ary Tree

- RSU: This entity serves as a gateway to provide OBUs within its transmission range with any requested information about revoked pseudonyms.

- OBU: Each vehicle is equipped with an OBU, which periodically broadcasts signed beacons that are received by neighbor OBUs and RSUs.

The proposed model is based on the use of a pseudonym $\mathrm{P}_{\mathrm{j}}$ set by each OBU, so that for each one the TTP provides the OBU with a corresponding private key $\mathrm{P}_{\mathrm{r}} \mathrm{P}_{\mathrm{j}}$. If any of those pseudonyms is revoked by the TTP, it inserts all the pseudonyms corresponding to the same $\mathrm{OBU}$ in the revocation tree. The TTP is also responsible for periodically updating the tree by deleting the expired pseudonyms, and for restructuring the tree when necessary. After each update, the TTP sends the corresponding modifications of the updated tree to all RSUs.

The RSU has to search vehicle pseudonyms in the revocation tree each time an OBU requests it. The RSU must provide the requesting OBU either with a verifiable revocation proof of any revoked pseudonym or with a signed message indicating that the requested pseudonym has not been revoked and is labelled as 'OK'. In the first case, by using the answer data, the OBU can verify the TTP signature of the received signed root, recompute the root of the revocation tree, and check it by comparing it with the received signed root.

The proposed scheme is computationally efficient since it obviates the need to sign each RSU reply, as it removes most of the trust from it. The only case when the RSU's trust is questioned is when it provides an 'OK' answer because that could be a fraud.
In this regard, when an $\mathrm{OBU}$ receives an 'OK' message signed by a cheating RSU, it trusts it momentarily. However, when it contacts another RSU, it asks it again about the same pseudonym. If this RSU provides the OBU with a proof of revocation whose timestamp contradicts the 'OK' answer signed by the questioned RSU, the OBU sends to the latter RSU an impeachment on the questioned RSU, so that the honest RSU can send it to the TTP who will revoke its public key by deleting it directly from the revoked RSU. Otherwise, if the second RSU also sends a signed 'OK' message, the OBU goes on asking about the same pseudonym until it reaches either a contradiction or a prefixed trust threshold.

Thus, each OBU stores locally in two separate and complementary structures, the pseudonyms of those OBUs that it has previously checked as unreliable, and of those OBUs that have been reliable till then. Therefore, in the future, if it reconnects with any of these vehicles, it can use such information to decide how to proceed. If there is no RSU nearby, it uses these data to decide whether to establish the communication or not. Otherwise, even if there is an RSU nearby, there is no need to re-ask it about a checked revoked pseudonym.

\section{Conclusions}

Efficient authentication of users in vehicular adhoc networks is one of the most difficult paradigms of the last years. Due to this reason, identity-based cryptography is here proposed to achieve certificateless authentication, which increases the efficiency and security of communications in 
vehicular networks. In addition, this paper uses Huffman codes in combination with authenticated dynamic hash k-ary trees to create a more efficient data structure. This structure allows using a tree where the most queried revoked pseudonyms correspond to the shortest paths in the tree. This provides a better model of the real behavior of vehicular networks, because some vehicles (such as public transport) are more common than others on the roads.

\section{Acknowledgements}

Research supported by the MINECO and the FEDER under Projects TIN2011- 25452 and IPT2012-0585-370000, and the scholarship BES-2012051817.

\section{References}

[1] G. Bertoni, J. Daemen, M. Peeters, G.V. Assche: "Keccak sponge function family main document", version 2.1, Updated submission to NIST (Round 2), 2010.

[2] S. Blake-Wilson: "Information security, mathematics, and public-key cryptography". Designs, Codes and Cryptography 19(2-3), pp. 77-99, 2000.

[3] D. Boneh, M. Franklin: "Identity-based encryption from the Weil pairing". Crypto. LNCS 2139, pp. 213-229, 2001.

[4] T. Cormen, C. Leiserson, R. Rivest: "Introduction to algorithms". MIT Press, 1990.

[5] ETSI: Intelligent Transport Systems. http://www.etsi.org/index.php/technologies-

clusters/technologies/intelligent-transport, Access Date: 02 Dec 2014.

[6] C. Ganan, J. Munoz, O. Esparza, J. Mata-Diaz, J. Alins: "Toward Revocation Data Handling Efficiency in VANETs". Communication Technologies for Vehicles. LNCS 7266, pp. 80-90, 2012.

[7] M. Goodrich, M. Shin, R. Tamassia, W. Winsborough: "Authenticated dictionaries for fresh attribute credentials". Trust Management. LNCS 2692, pp. 332-347, 2003.

[8] M. Goodrich, R. Tamassia, N. Triandopoulos, R. Cohen: "Authenticated data structures for graph and geometric searching". CT-RSA. LNCS 2612, pp. 295-313, 2003 .

[9] J.P. Hubaux, S. Capkun, J. Luo: "The security and privacy of smart vehicles". IEEE Security and Privacy 2(3), pp. 49-55, 2004

[10] D. Huffman: "A Method for the Construction of Minimum-Redundancy Codes". Proceedings of IRE 40 (9), pp. 1098-1101, 1952.

[11] IEEE-1609: "Family of standards for Wireless Access in Vehicular Environments" (WAVE). U.S. Department of Transportation, 2006.
[12] M. Jakobsson, T. Leighton., S. Micali, M. Szydlo: "Fractal merkle tree representation and traversal". CTRSA. LNCS 2612, pp.314-326, 2003.

[13] M. Jakobsson, S. Wetzel: "Efficient attribute authentication with applications to ad hoc networks". ACM workshop on vehicular ad hoc networks, pp. 38-46, 2004.

[14] A. Joux: "The weil and tate pairings as building blocks for public key cryptosystems". Algorithmic Number Theory Symposium. LNCS 2369, pp. 20-32, 2002.

[15] P. Kocher: "On certificate revocation and validation". FC'98. LNCS 1465, pp. 172-177, 1998.

[16] R. Merkle: "Protocols for public key cryptosystems". IEEE Security and privacy 1109, pp. 122-134, 1980.

[17] V. Miller: "Short programs for functions on curves". Unpublished manuscript, 97, pp. 101-102, 1986.

[18] J. Muñoz, J. Forne, O. Esparza, J. Manel: "Efficient Certificate Revocation System Implementation: Huffman Merkle Hash Tree (HuffMHT)." TrustBus, pp 119-127, 2005.

[19] M. Raya, J.P. Hubaux: "Securing vehicular ad hoc networks". Computer Security 15(1), pp. 39-68, 2007.

[20] A. Shamir: "Identity-based cryptosystems and signature schemes". Crypto. LNCS 196, pp. 47-53, 1985. 